\title{
Autoavaliação da saúde e qualidade da dieta: comparação em idosos hospitalizados
}

\author{
Greiskelly Didur Klossniak; Maria Vitória Matos"*; Diana Souza Santos Vaz"*; Dalton Luiz Schiessel ${ }^{* * * *}$;

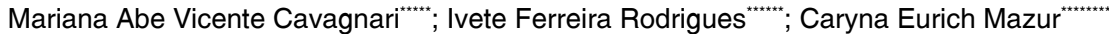

\section{Resumo}

O estudo objetiva associar a qualidade da dieta e autoavaliação da saúde de idosos hospitalizados. Estudo transversal, realizado em um hospital de Guarapuava/PR, com 100 idosos. Foi aplicado um questionário socioeconômico, que também continha perguntas para auto percepção da saúde, recordatório alimentar habitual para mensurar a qualidade da dieta (IQD) de cada indivíduo, cujo foi calculado por meio de índice próprio. Para associação foi utilizado o teste exato de Fisher, correlação e análise descritiva. A média de idade foi de $72,39 \pm 7,77$ anos, sendo $50 \%$ do gênero feminino. A autoavaliação sobre a qualidade da dieta, demonstrou que há necessidade de modificação (52\%). A maioria $(41 \%)$ classificou seu estado de saúde como ruim. Não houve associação entre o IQD e a autoavaliação da saúde $(p>0,05)$, demonstrando que a percepção de saúde não interfere na qualidade da dieta. Portanto, não houve associação entre a auto percepção da saúde e a qualidade da dieta em idosos hospitalizados.

Palavras-chave: Dieta. Longevo. Nutrição. Saúde do idoso.

\section{Introdução}

O envelhecimento está associado ao acúmulo de danos moleculares e celulares, e mudanças que vão além das perdas biológicas (LARSSON et al., 2019). Com o tempo, esses danos levam a uma perda gradual nas reservas fisiológicas e um aumento do risco de contrair diversas doenças e aumentar a morbimortalidade nessa população (OMS, 2015).

Sendo assim, aos 60 anos de idade, os indivíduos possuem maiores chances de serem afetados por doenças não transmissíveis, incluindo doenças cardíacas,

* Nutricionista. Universidade Estadual do Centro-Oeste, Guarapuava/PR. E-mail: greiskellyd@gmail.com.

** Nutricionista. Universidade Estadual do Centro-Oeste, Guarapuava/PR. E-mail: matosmvitoria@gmail.com.

**** Nutricionista. Doutoranda em Ciências da Saúde pela Universidade Estadual de Campinas, Campinas/SP. Mestrado em Promoção da Saúde pelo Centro de Ensino Superior de Maringá/PR. E-mail: nutridianavaz@ gmail.com.

***** Nutricionista. Doutorado em Biologia Celular e Molecular pela Universidade Federal do Paraná, Curitiba/PR. Pós-doutorado no Cross Cancer Institute da University of Alberta, Edmonton, Canadá. Curso de Nutrição da Universidade Estadual do Centro-Oeste, Guarapuava/PR. E-mail: daltonls68@gmail.com.

****** Nutricionista. Universidade Estadual do Centro-Oeste, Guarapuava/PR. E-mail: marianaav@hotmail.com.

******* Nutricionista. Especialização em Nutrição Clínica pela Universidade Norte do Paraná. Associação de Saúde Frederico Guilherme Virmond, Guarapuava/PR. E-mail: ivete@ivirmond.com.br.

******** Nutricionista. Mestrado em Segurança Alimentar e Nutricional e doutorado em Medicina Interna e Ciências da Saúde pela Universidade Federal do Paraná, Curitiba/PR. Departamento de Nutrição da Universidade Estadual do Centro-Oeste, Guarapuava/PR. E-mail: carynanutricionista@gmail.com.

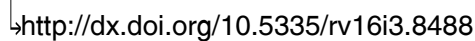


acidente vascular cerebral, doenças respiratórias crônicas, câncer e demências. Existem outras comorbidades crônicas, como a fragilidade (diminuição de massa e força muscular, alteração de marcha e equilíbrio, exaustão, perda de equilíbrio, etc.), que pode ter uma prevalência em torno de $10 \%$ em pessoas com idade superior a 65 anos ou agudas como por exemplo, delírio, que pode resultar de várias determinantes tão distintas como uma infecção ou efeitos colaterais de uma cirurgia (OMS, 2015; LARSSON et al., 2019).

Durante o processo do envelhecimento, várias alterações fisiológicas acontecem no organismo: diminuição da elasticidade dos tecidos e da massa muscular, perda de líquido intracelular, decréscimo da estatura além de mudanças na quantidade e distribuição do tecido muscular subcutâneo, ocorre redução deste tecido nos membros e há um progressivo acúmulo de tecido adiposo na cavidade abdominal (LARSSON et al., 2019; OIKAWA; HOLLOWAY; PHILLIPS, 2019). Além disso, alterações fisiológicas que limitam o repertório alimentar, seja pelas restrições decorrentes de enfermidades crônicas ou das alterações de mastigação e digestão, como com as condições de mobilidade, autonomia, independência financeira, condições de saúde e da composição familiar. Esses são fatores decisivos sobre o repertório alimentar, a frequência e a qualidade da alimentação dos idosos (GRANIC; SAYER; ROBINSON, 2019).

Segundo o Instituto Brasileiro de Geografia e Estatística (IBGE) conforme censo demográfico de 2010, a população idosa correspondia à $7,4 \%$, sendo as regiões Sudeste e Sul com maior proporção de idosos. Em Guarapuava, Paraná, no mês de junho de 2016 ocorreram cerca de 226 internações de idosos, sendo por causas gerais não classificando por tipos de doenças (BRASIL, 2016).

Ter conhecimentos sobre a dieta pregressa e a qualidade da dieta do idoso e sua auto percepção da saúde se faz necessário para que sejam traçadas estratégias de intervenção nutricional e melhora da atuação da equipe multidisciplinar, tendo em vista também a melhora da qualidade de vida e do prognóstico do idoso com doenças crônicas.

Dessa forma, o objetivo do presente trabalho foi associar a qualidade da dieta e autoavaliação da saúde de idosos internados em um hospital de Guarapuava, Paraná.

\section{Métodos}

Trata-se de um estudo transversal com amostra por conveniência, que incluiu 100 pacientes idosos hospitalizados pelo Sistema Único de Saúde (SUS) em um hospital de Guarapuava, Paraná. Não fizeram parte desse estudo pacientes com membros amputados e que estavam impossibilitados de comunicação. Os critérios de inclusão foram idosos internados com qualquer diagnóstico clínico, no período de junho a agosto de 2016. Este estudo foi aprovado pelo Comitê de Ética em Pesquisa da Universidade Estadual do Centro-Oeste, CAAE número 55903216.5.0000.0106. 
Os pacientes convidados a participar assinaram o Termo de Consentimento Livre e Esclarecido concordando com a participação no estudo.

Os dados foram coletados por duas pesquisadoras previamente treinadas e calibradas, afim de obter fidedignidade dos dados. Foi aplicado um questionário socioeconômico elaborado para pesquisa, contendo questões sobre: sexo, faixa etária, anos de estudo, renda familiar, número de pessoas que residiam no domicílio, número de medicamentos administrados, se o paciente fumava e se ingeria bebidas alcoólicas. Em relação ao diagnóstico clínico, o mesmo foi coletado no prontuário do paciente. Foi ainda questionada a aceitação da dieta hospitalar. Outros dados coletados foram: a prática de atividade física, se o paciente foi hospitalizado no último ano e o número de vezes. Para autoavaliação de saúde foram elaboradas para esse estudo variáveis categóricas o qual o paciente relatava que sua saúde era: "excelente", "muito boa", "ruim", e "muito ruim".

Foi utilizado o recordatório alimentar habitual (RAH), como instrumento de avaliação dietética, no qual o paciente ou seu acompanhante relatou os alimentos e respectivas porções que eram habitualmente consumidos por ele durante um dia em seu domicílio. Para o cálculo de Índice de qualidade da dieta (IQD) foi utilizado o aplicativo Nutrabem 2.0®. Para avaliação do IQD foi utilizado parâmetro preconizado por Fisberg et al. (2004). A pontuação do índice é obtida por dez componentes característicos de uma dieta saudável, cada um deles é pontuado de zero a dez. O valor máximo é de cem pontos. Para efeitos de análise foi considerado uma dieta inadequada abaixo de 51 pontos; uma dieta que necessita modificação de 51 a 80 pontos; e uma dieta saudável acima de 80 pontos (FISBERG et al., 2004). O instrumento leva em consideração os grupos de alimentos (cereais, pães, tubérculos e raízes; verduras e legumes; frutas; leite e produtos lácteos; carnes, ovos e feijão), gordura total, gordura saturada, colesterol da dieta e variedade da dieta (FISBERG et al., 2004).

Os idosos foram pesados em balança digital com capacidade de $150 \mathrm{~kg}$ e a estatura foi aferida em parede sem rodapés com auxílio de uma fita métrica inelástica. $\mathrm{O}$ índice de massa corporal (IMC), foi calculado por meio da divisão do peso em quilogramas $(\mathrm{kg})$ pelo quadrado da estatura $(\mathrm{m})$ e o resultado expresso em $\mathrm{kg} / \mathrm{m}^{2}$. Para classificação do IMC foi utilizado critérios preconizados por Lipschitz (1994).

Para análise estatística os dados foram organizados em planilha e após procedeu-se analise descritiva dos dados (frequências, médias, desvio-padrão, mediana, mínimo e máximo). Foi utilizado o teste de Kolmogorov-Smirnov para determinação de normalidade da amostra. A comparação das variáveis categóricas foi realizada por meio do teste exato de Fisher. Para associação do IQD com outras variáveis foram utilizadas as correlações de Pearson e Spearmann, de acordo com a distribuição de normalidade. A análise foi realizada com auxílio do 
software SPSS versão 22.0, com nível de significância de $5 \%(\mathrm{p}<0,05)$.

\section{Resultados}

Participaram do estudo 100 idosos, com média de idade de 72,39 $\pm 7,77$ anos, sendo $50 \%$ do sexo feminino. A média de renda familiar foi de 1.594,38 $\pm 930,19$ reais. A maioria ( $81 \%)$ apresentava menos de quatro anos de estudo. $\mathrm{O}$ diagnóstico clínico mais presente na internação (36\%) foi de doenças pulmonares (Pneumonia, Doença Pulmonar Obstrutiva Crônica, Bronquite), 24\% dos idosos eram tabagistas (Tabela 1

) .

Tabela 1 - Perfil socioeconômico e de saúde de idosos hospitalizados. Guarapuava, 2016. ( $\mathrm{n}=100)$.

\begin{tabular}{|l|l|}
\hline \multicolumn{1}{|c|}{ Variáveis } & $\mathbf{n}$ \\
\hline Sexo & \\
\hline Feminino & 50 \\
\hline Masculino & 50 \\
\hline Faixa etária & \\
\hline De 60 a 69 anos & 39 \\
\hline De 70 a 79 anos & 43 \\
\hline 80 ou mais anos & 18 \\
\hline Anos de estudo & \\
\hline Menos que 4 anos & 81 \\
\hline De 4 a 10 anos & 18 \\
\hline Mais que 10 anos & 1 \\
\hline Diagnóstico clínico & \\
\hline Pulmonar & 36 \\
\hline Ósseo-muscular & 13 \\
\hline Hepáticas & 12 \\
\hline Cardiovasculares & 11 \\
\hline Outros & 28 \\
\hline
\end{tabular}

Nota: * SM = Salário mínimo (R\$ 880,00 referente ao mês de agosto/2016).

A aceitação da dieta hospitalar foi considerada boa por $69 \%$ dos idosos. Entretanto a qualidade da dieta, mensurada pelo IQD, mostrou que há necessidade de modificação em $52 \%$ da amostra e a maioria (56\%) classificou o estado de saúde como ruim/muito ruim, como demonstrado no Figura 1. 
Figura 1 - Percentuais de aceitação e qualidade da dieta e auto percepção da saúde de idosos hospitalizados. Guarapuava, $2016(\mathrm{n}=100)$.

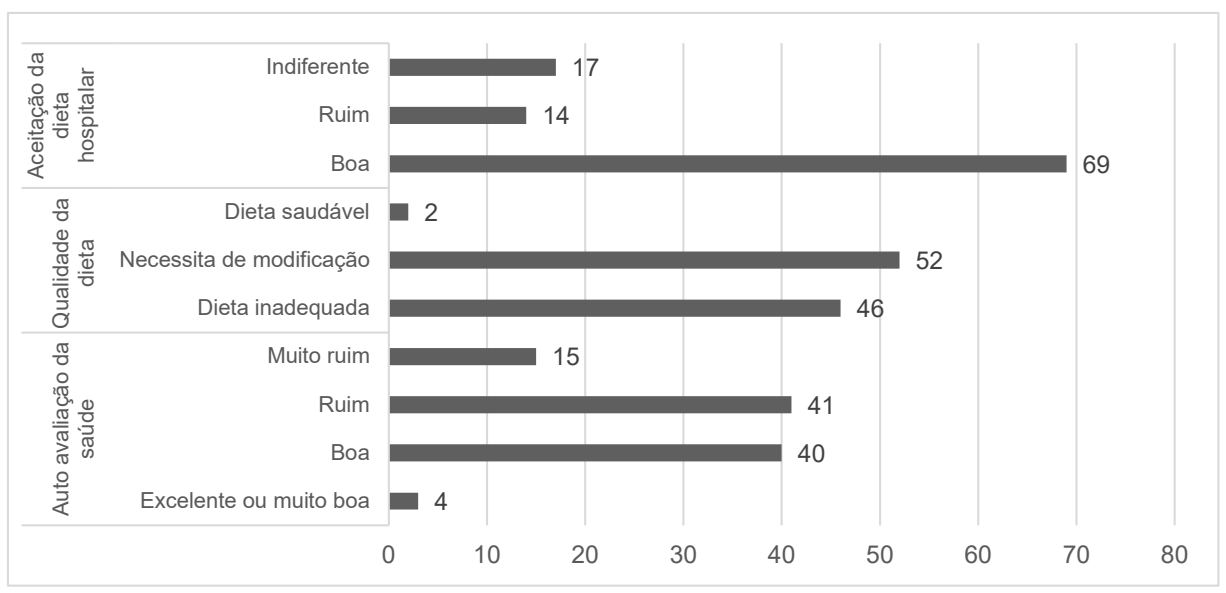

Conforme apresentado na Tabela 2, sua percepção de saúde com ruim e muito os pacientes que relataram reinterna- $\operatorname{ruim}(\mathrm{p}<0,05)$. ções hospitalares anteriores referiram

\begin{tabular}{|l|c|c|c|}
\hline \multirow{2}{*}{ Variáveis } & \multicolumn{2}{|c|}{ Autoavaliação da Saúde } & \multirow{2}{*}{} \\
\cline { 2 - 3 } & Boa/Excelente & Ruim/Muito ruim & \\
\hline Sexo & & & \\
\hline Feminino & 18 & 32 & 0,080 \\
\hline Masculino & 26 & 24 & \\
\hline Diagnósticos clínicos & & & \\
\hline Pulmonar & 11 & 25 & \\
\hline Ósseo-muscular & 7 & 6 & 0,280 \\
\hline Hepáticas & 6 & 6 & \\
\hline Cardiovasculares & 7 & 4 & 0,030 \\
\hline Outros & 13 & 15 & \\
\hline Internações no último ano & & & \\
\hline Uma duas & 41 & 43 & \\
\hline Três ou mais & 3 & 13 & \\
\hline Prática de atividade física & & & \\
\hline Sim & 3 & 54 & \\
\hline Não & 41 & & \\
\hline
\end{tabular}




\begin{tabular}{|c|c|c|c|}
\hline \multirow[t]{2}{*}{ Variáveis } & \multicolumn{2}{|c|}{ Autoavaliação da Saúde } & \multirow[t]{2}{*}{$\mathrm{p}$} \\
\hline & Boa/Excelente & Ruim/Muito ruim & \\
\hline Magreza & 22 & 23 & \\
\hline Eutrofia & 15 & 20 & 0,480 \\
\hline Excesso de peso & 7 & 13 & \\
\hline \multicolumn{4}{|l|}{ Etilismo } \\
\hline Sim & 6 & 2 & 0,130 \\
\hline Não & 38 & 54 & \\
\hline \multicolumn{4}{|l|}{ Tabagismo } \\
\hline Sim & 10 & 14 & 0,820 \\
\hline Não & 34 & 42 & \\
\hline \multicolumn{4}{|l|}{ Qualidade da dieta } \\
\hline Dieta inadequada & 18 & 28 & \\
\hline Necessita de modificação & 25 & 27 & 0,660 \\
\hline Dieta saudável & 1 & 1 & \\
\hline
\end{tabular}

Nota: Significativo para $\mathrm{p}<0,05$; Teste exato de Fisher.

A dieta considerada inadequada de acordo com IQD perfez $46 \%$ da amostra. Os cereais integrais foram pouco consumidos, assim como as frutas. Já as oleaginosas não foram relatadas por nenhum dos idosos. Os alimentos mais consumidos foram cereais refinados, carne bovina, suína e hortaliças (Tabela 3).

Tabela 3 - Variáveis descritivas referentes às porções de Índice de Qualidade da Dieta (IQD) de idosos hospitalizados. Guarapuava, $2016(\mathrm{n}=100)$.

\begin{tabular}{|l|l|l|l|}
\hline \multicolumn{1}{|c|}{ Variáveis } & \multicolumn{1}{c|}{ Média } & \multicolumn{1}{c|}{ Desvio-padrão } & \multicolumn{1}{c|}{ Mediana } \\
\hline Aves, pescados e ovos & 1,47 & 1,96 & 0,96 \\
\hline $\begin{array}{l}\text { Cereais e pães integrais, tubér- } \\
\text { culos e raízes }\end{array}$ & 0,41 & 0,93 & 0,00 \\
\hline Frutas & 0,57 & 0,96 & 0,00 \\
\hline Hortaliças & 8,41 & 10,56 & 5,35 \\
\hline Leguminosas & 1,49 & 1,30 & 1,30 \\
\hline Leite e derivados & 0,75 & 0,76 & 0,69 \\
\hline Oleaginosas & 0,00 & 0,00 & 0,00 \\
\hline Óleos vegetais & 1,40 & 0,94 & 1,22 \\
\hline Açúcares e doces & 0,92 & 1,58 & 0,37 \\
\hline Carnes bovina e suína & 2,22 & 2,91 & 0,46 \\
\hline Cereais e pães refinados & 2,81 & 2,23 & 2,16 \\
\hline
\end{tabular}




\begin{tabular}{|l|l|l|l|}
\hline \multicolumn{1}{|c|}{ Variáveis } & \multicolumn{1}{c|}{ Média } & \multicolumn{1}{c|}{ Desvio-padrão } & \multicolumn{1}{c|}{ Mediana } \\
\hline Gorduras de origem animal & 0,02 & 0,10 & 0,00 \\
\hline IQD - Pontuação total & 51,47 & 13,48 & 53,17 \\
\hline
\end{tabular}

De acordo com as correlações observadas no quadro 1 , quanto maior a qualidade da dieta maior é o IMC do paciente $\left(r^{2}=0,980 ; p<0,05\right)$. Houve uma tendência estatística quando se associa a renda familiar com o IQD ( $\mathrm{p}=$ 0,070 ) porém com uma correlação fraca $\left(\mathrm{r}^{2}=0,180\right)$. Quando comparada com a autoavaliação da saúde a correlação foi fraca, inversa e sem significância $\left(\mathrm{r}^{2}=\right.$ $-0,070 ; p>0,05$ ). Já, a idade do paciente, anos de estudo, número de moradores no domicílio, internações no último ano e medicamentos utilizados não tiveram boa correlação com o IQD.

Tabela 4 - Coeficiente de determinação (r2) e p valor entre o índice de qualidade da dieta (IQD) e fatores associados em idosos hospitalizados. Guarapuava, 2016 ( $n=100$.

\begin{tabular}{|l|c|c|}
\hline \multicolumn{1}{|c|}{ Variáveis } & $\mathbf{r}^{\mathbf{2}}$ & $\mathbf{p}$ \\
\hline Idade* $^{*}$ & 0,980 & 0,870 \\
\hline Renda familiar $^{*}$ & 0,180 & 0,070 \\
\hline Autoavaliação da saúde** & $-0,070$ & 0,510 \\
\hline Anos de estudo* & $-0,060$ & 0,580 \\
\hline $\begin{array}{l}\text { Número de moradores no do- } \\
\text { micílio* }\end{array}$ & 0,090 & 0,340 \\
\hline Internações no último ano* $^{*}$ & $-0,210$ & 0,030 \\
\hline Medicamentos utilizados $^{*}$ & 0,010 & 0,270 \\
\hline IMC $^{*}$ & 0,980 & 0,020 \\
\hline
\end{tabular}

Nota: *Relativo à correlação de Pearson; *^Relativo à correlação de Spearmann.

\section{Discussão}

Nesta pesquisa foi constatada média de idade de, aproximadamente, 72 anos, sendo metade do sexo feminino. As doenças pulmonares foram o diagnóstico clínico mais presente, seguida pelas doenças ósseo-musculares. A qualidade da dieta, mensurada pelo IQD, mostrou que há necessidade de modificação em pouco mais da metade da amostra. A maioria dos idosos classificou seu estado de saúde como ruim. A dieta foi considerada inadequada de acordo com IQD perfez parte da amostra. Notou-se que os cereais integrais foram pouco consumidos, assim como as frutas.

Dessa forma, sabe-se que o envelhecimento da população apresenta características diferentes de acordo com as condições de vida de cada país e região. No geral, as condições necessárias para alcançar a longevidade melhoraram no mundo inteiro, levando a um aumento 
da população considerada na terceira idade (COSTA; MATIAS, 2005). Quando se trata de idosos mais velhos, o estrato formado por indivíduos com 75 anos ou mais de idade apresenta maior crescimento relativo $(49,3 \%)$ nos últimos dez anos, quando comparado ao total da população idosa (BRASIL, 2009).

A média de idade dos idosos participantes do estudo foi mais de 70 anos, em 1940, a esperança de vida do brasileiro sequer atingia os 50 anos de idade (45,5 anos) (BRASIL, 2009). Os avanços no setor da saúde e as melhorias nas condições gerais de vida da população contribuíram para que, 71 anos mais tarde, este indicador atingisse 74,08 anos, em 2011, próximo a idade média dos idosos estudados. Segundo a projeção de população do IBGE, em 2050 esse indicador deverá chegar aos 81,3 anos (BRASIL, 2009).

Embora com o aumento da longevidade, a pessoa idosa corre maior risco de passar a viver com enfermidades crônicas por um longo período, o que pode comprometer sua qualidade de vida. Essa condição exige políticas públicas de atenção que possibilitem a oferta de cuidados continuados e integrados às pessoas idosas (GONÇALVES et al., 2006; LEITE, 2009; LARSSON et al., 2019).

Houve uma homogeneidade da amostra estudada em relação ao sexo. Porém, pesquisas têm sugerido que as taxas de mortalidade mais elevadas dos homens em idades mais jovens poderiam permitir que, na velhice, a composição do grupo etário masculino fosse mais favo- rável que o feminino (PERLS; KUNKEL; PUCA, 2002; OIKAWA; HOLLOWAY; PHILLIPS, 2019). Isto resultaria, diferentemente do apresentado nesta pesquisa, num grupo heterogêneo no qual haveria um número maior de idosas mais fragilizadas e susceptíveis do que idosos (CAMARGO; PERPÉTUO; MACHADO, 2005; CAMARGO; RODRIGUES; MACHADO, 2009; LARSSON et al., 2019). Além disso, sugere-se que as condições de saúde das mulheres podem ser reflexo de condições econômicas, sociais e culturais desiguais, nos diversos momentos de suas vidas (GOLDANI, 1999; BARRETO et al., 2002).

Grande parte dos idosos estudados apresentava baixa escolaridade (menos de quatro anos de estudo). Lima e Bueno (2009) encontraram um número reduzido de anos de ensino formal frequentado pelos idosos. Os autores sugerem a relação com a realidade pregressa de quem hoje conta com 80 ou mais anos de idade, em que o acesso à educação era difícil para essa população.

No presente estudo houve predominância das doenças pulmonares. Sabe-se que entre as causas de doença e óbito em idosos, a doença pulmonar obstrutiva crônica se destaca devido à sua alta prevalência e caráter progressivo. As doenças pulmonares podem ter impacto negativo na alimentação, especialmente pela inapetência e dispneia, sintomatologia frequente nesses pacientes, sobretudo quando essas doenças cronificam, tais sinais e sintomas tornam-se comuns, prejudicando a nutrição e que por consequência acabam depletando o estado 
nutricional do idoso (PATTERSON et al., 2017). A infecção respiratória também vem sendo apontada como uma das principais causas de morbimortalidade entre os idosos, podendo estar associada à exacerbação clínica da doença pulmonar obstrutiva crônica (NICHOLSON et al., 1997; PATTERSON et al., 2017). Segundo dados do SUS (BRASIL, 2016) o número de interações hospitalares de idosos devido ao diagnóstico doenças pulmonares em Guarapuava/PR foi de 70 pacientes no mês de junho de 2016 . No presente estudo foi obtida $36 \%$ da amostra com o mesmo diagnóstico.

A autoavaliação de saúde constitui-se em um interessante preditor de morbidade, mortalidade e declínio funcional, e vem sendo frequentemente utilizada em pesquisas direcionadas aos idosos, por revelar a percepção integrada do indivíduo envolvendo as dimensões biológica, psicológica e social (GILTAY; VOLLAARD; KROMHOUT, 2012).

A maioria dos idosos (56\%) auto avaliou sua saúde como ruim/muito ruim. Há evidência de que a autoavaliação da saúde ruim ou muito ruim, se associa a diversos fatores como: sedentarismo, baixo peso e obesidade, doenças crônicas, maior uso de medicamentos, e incapacidade funcional. Estudo de Moraes, Moreira e Luiz (2011) também demonstrou que a autoavaliação piora com a idade, contudo esta relação não foi avaliada no presente estudo.

Também em estudo com idosos, Cardoso et al. (2014) encontraram 30,9\% de avaliações como ruim ou péssima, estando muito próximo ao presente estudo. Neste mesmo estudo o uso de medicamentos também se mostrou associado a autoavaliação do estado de saúde ruim/ péssima. Sendo o consumo médio de medicamentos maior para quem identificou-se neste tipo de avaliação (SILVA et al., 2012). No presente estudo $80 \%$ da amostra faz uso de dois a três medicamento ao dia.

As reinterações hospitalares foram associadas com a percepção de saúde ( $\mathrm{p}$ $<0,05)$. Sabe-se que, devido às questões biopsicossociais o idoso tem declínio cognitivo e quanto maior o número de medicamentos utilizados diariamente e a dependência em serviços de saúde, maior sua culpa diante de situações, como doenças recorrentes. Com isso, percebe-se que a percepção de saúde fica deteriorada, assim como, provavelmente, ocorreu no presente estudo.

A maioria dos idosos relatou ter uma boa aceitação da dieta hospitalar, apesar da maior parte não ter boa qualidade nutricional da alimentação consumida no domicílio. É importante lembrar que o IQD foi determinado a partir de instrumento de avaliação alimentar a longo prazo, estabelecido a partir do recordatório alimentar habitual. Nesse sentido, mensurar a qualidade da dieta torna-se importante nessa população, pois presume-se que os idosos, devido as alterações fisiológicas, podem ter uma alimentação deficitária com baixa qualidade nutricional.

Assumpção et al. (2014) em trabalho conduzido em Campinas, encontraram um escore médio do IQD de 62 pontos. No Município de São Paulo, os idosos 
avaliados no ano de 2008 apresentaram 62,8 pontos, valor que é bastante próximo ao observado em Campinas (ANDRADE, 2013). No presente estudo a pontuação média encontrada foi de 51,47 pontos, sinalizando dieta que precisa de modificação e que há muito a avançar na melhoria da qualidade da alimentação desses idosos. Entre os pontos para melhorar essa alimentação encontram-se: aumentar o consumo de cereais integrais, frutas e oleaginosas. Esses alimentos são importantes pois regulam a função intestinal, o controle glicêmico e pressórico, além de possuírem antioxidantes fundamentais principalmente para as respostas imunológica e inflamatória do organismo.

Os resultados vão de encontro com os de Assumpção et al. (2014) que também indicaram piores pontuações nos componentes de cereais integrais, sódio, leite e derivados, frutas totais e frutas integrais. Juan et al. (2008) e Hiza et al. (2012) ao analisarem idosos com 65 anos ou mais incluídos no National Health and Nutrition Examination Survey, também encontraram escores baixos para grãos integrais, sódio e leite, no entanto a pontuação de frutas totais foi bastante elevada.

Dados da Vigilância de Fatores de Risco e Proteção para Doenças Crônicas por Inquérito Telefônico demostram que a ingestão de frutas e hortaliças tende a aumentar com a idade, mas ainda é inferior ao mínimo de $400 \mathrm{~g} / \mathrm{dia}$ recomendado pela Organização Mundial da Saúde (OMS, 2015). A presente pesquisa apresentou correlação entre IMC e IQD, quanto maior o IMC do paciente melhor a qualidade da dieta, podendo ser decorrente da presença de doenças crônicas não transmissíveis - geralmente comuns na faixa etária estudada, estas tendem aumentar a preocupação do idoso em relação à sua dieta, melhorando assim a sua qualidade e, provavelmente, aumentando seu peso corporal.

A limitação do estudo foi que no período de coleta de dados, a cidade se encontrava em inverno intenso, sendo os idosos mais propensos às doenças $\mathrm{e}$ com diagnósticos clínicos similares devido ao clima. Além disso cabe colocar que o recordatório alimentar habitual não é o melhor método para avaliar o consumo alimentar. O questionário de autopercepção de saúde não é validado, isso também pode ser considerada uma limitação potencial do estudo.

Conclusão

A ingestão alimentar não interfere na autoavaliação de saúde de idosos hospitalizados. A autoavaliação da saúde foi considerada ruim e a dieta habitual necessita de modificações. Os idosos encontravam-se hospitalizados devido a doenças pulmonares e maioria apresentava magreza, não houve associação da autoavaliação da saúde com as demais variáveis do estudo. Há aplicabilidade do questionário de autoavaliação da saúde e sua associação com a dieta habitual, por meio desses dados pode-se promover um cuidado de saúde holístico e integral ao paciente, especialmente ao idoso. Sugerem-se outros trabalhos com esta temática, com maior número amostral a 
fim de confirmar ou não a relação entre dieta e a percepção da saúde.

\section{Self- rated health and diet quality: comparison elderly hospitalized}

\section{Abstract}

This study aim associate diet quality and self-assessment of hospitalized elderly. Cross-sectional study in a hospital in Guarapuava/PR, Brazil, with 100 elderly. A socioeconomic questionnaire was applied, which also included questions for self-perception of health, the usual food day was used to measure the quality of the diet (QDI) of each individual, which was calculated by means of their own index. Fisher's exact test, correlation and descriptive analysis were used for association. The mean age was $72.39 \pm 7.77$ years, being $50 \%$ female. Self-assessment of diet quality, measured by QDI, showed that there is a need for modification (52\%). The majority $(41 \%)$ rated their health status as poor. There was no association between QDI and self-rated health $(p>0.05)$, demonstrating that health perception does not interfere with diet quality. There was no association between self-perceived health and diet quality among hospitalized elderly.

Keywords: Diet. Elderly. Nutrition. Health.

\section{Referências}

ANDRADE, S. C. Mudanças na qualidade da dieta e seus fatores associados em residentes do Município de São Paulo em 20032008: estudo de base populacional [Tese de Doutorado]. São Paulo: Universidade de São Paulo, 2013.
ASSUMPÇÃO, D. D. et al. Qualidade da dieta e fatores associados entre idosos: estudo de base populacional em Campinas, São Paulo, Brasil. Caderno de Saúde Pública, Rio de Janeiro, v. 30, n. 8, p. 1680-1694, 2014.

BARRETO, S. M. et al. Gênero e desigualdades em saúde entre idosos brasileiros. In: Anais da Oficina de Trabalho sobre Desigualdades Sociais e de Gênero em Saúde de Idosos no Brasil. Ouro Preto: Ministério da Saúde, Fundação Oswaldo Cruz, Universidade Federal de Minas Gerais, 2002. p. 59-69.

BRASIL. Instituto Brasileiro De Geografia e Estatística - IBGE. Em 2008, esperança de vida dos brasileiros chega a 72,86 anos. 2009. Disponível em: <http://www.ibge.gov. br/home/presidencia/noticias/noticia_visualiza.php?id_noticia=1507 \&id_pagina $=1>$. Acesso em: 16 ago. 2016.

BRASIL. Ministério da Saúde. Departamento de Informática do SUS (DATASUS). Disponível em: <http://www.datasus.gov.br/DATASUS/index.php?area $=0203 \& i d=6927 \& V O b-$ $\mathrm{j}=\mathrm{http} / /$ tabnet.datasus.gov.br/cgi/deftohtm. exe?sih/cnv/nr>. Acesso em: 24 ago. 2016.

CAMARGO, M. C. S. et al. Expectativa de vida com incapacidade funcional em idosos em São Paulo, Brasil. Revista Panamericana de Salud Publica, v. 17, n. 5-6, p. 379-386, 2005.

CAMARGO, M. C. S. et al. Expectativa de vida saudável para idosos brasileiros, 2003. Ciência e Saúde Coletiva, Rio de Janeiro, v. 14, n. 5, p. 1903-1909, 2009.

CARDOSO, J. D. C. et al. Autoavaliação de saúde ruim e fatores associados em idosos residentes em zona urbana. Revista Gaúcha de Enfermagem, Porto Alegre, v. 35, n. 4, p. 35-41, 2014.

COSTA, H. O.; MATIAS, C. O impacto da voz na qualidade da vida da mulher idosa. Revista Brasileira de Otorrinolaringologia, São Paulo, v. 71, n. 2, p. 172-178, 2005.

FISBERG, R. M. et al. Índice de Qualidade da Dieta: avaliação da adaptação e aplicabi- 
lidade. Revista de Nutrição, Campinas, v. 17, n. 3, p. 301-318, 2004.

GRANIC, A.; SAYER, A. A.; ROBINSON, S. M. Dietary patterns, skeletal muscle health, and sarcopenia in older adults. Nutrients, v. 11, n. 4, pii. E745, 2019.

GILTAY, E. J. et al. Self-rated health and physician-rated health as independent predictors of mortality in elderly men. Age Ageing, v. 41, p. 165-71, 2012. DOI: 10.1093/ ageing/afr161

GOLDANI, A. M. Mulheres e envelhecimento: desafios para novos contratos intergeracionais e de gênero. In: CAMARANO, A. A. organizadora. Muito além dos 60: os novos idosos brasileiros. Rio de Janeiro: IPEA, 1999. p. 75-113.

GONÇALVES, L. H. T. et al. Perfil da família cuidadora de idoso doente/ fragilizado do contexto sociocultural de Florianópolis, SC. Texto \& Contexto em Enfermagem, Florianópolis, v. 15, n. 4, p. 570-577, 2006.

HIZA, H. A. B. et al. Diet quality of Americans differs by age, sex, race/ ethnicity, income, and education level. Journal of the Academy of Nutrition and Dietetics, v. 113, n. 2, p. 297-306, 2012.

JUAN, W. Y. et al. Diet quality of older Americans in 1994-96 and 2001-02 as measured by the Healthy Eating Index-2005. Washington DC: Center for Nutrition Policy and Promotion, U.S. Department of Agriculture, 2008.

LARSSON, L.; DEGENS, H.; LI; M.; SALVIATI, L.; LEE, Y.I.; THOMPSON, W. Sarcopenia: Aging-Related Loss of Muscle Mass and Function. Physiological Reviews, v. 99, n. 1, p. 427-511, 2019.

LEITE, M. T. Envelhecimento humano: novas e velhas demandas no campo da saúde. In: DALLEPIANE, L. B. organizador. Envelhecimento humano: campo de saberes e práticas em saúde coletiva. Ijuí/RS: Unijuí, 2009. p. 95-106.
LIMA, L. C. V.; BUENO, C. M. L. B. Envelhecimento e gênero: a vulnerabilidade de idosas no Brasil. Revista Saúde e Pesquisa, Maringá, v. 2, n. 2, p. 273-280, 2009.

LIPSCHITZ, D. A. Screening for nutritional status in the elderly. Primary Care, v. 21, n. 1, p. 55-68, 1994.

MORAES, J. R. et al. Associação entre o estado de saúde autorreferido de adultos e a área de localização do domicílio: uma análise de regressão logística ordinal usando a PNAD 2008. Ciência e Saúde Coletiva, Rio de Janeiro, v. 16, n. 9, p. 3769-3780, 2011.

OIKAWA, S. Y.; HOLLOWAY, T. M.; PHILLIPS, S. M. The Impact of Step Reduction on Muscle Health in Aging: Protein and Exercise as Countermeasures. Frontiers in Nutrition, v. 24, n. 6, p. 75, 2019.

ORGANIZAÇÃO MUNDIAL DASAÚDE. $R e$ sumo: Relatório Mundial de Envelhecimento e Saúde, 2015.

ORGANIZAÇÃO PAN-AMERICANA DA SAÚDE. Saúde nas Américas: 2007. Washington DC: Organização Pan-Americana da Saúde, 2007.

PATTERSON, K. C.; SHAH, R. J.; PORTEOUS, M. K.; CHRISTIE, J. D.; D'ERRICO, C. A.; CHADWICK, M. et al. Interstitial Lung Disease in the Elderly. CHEST Journal - American College of Chest Physicians, v. 151, n. 4, p. 838-844, 2017.

PERLS, T. et al. The genetics of exceptional human longevity. Journal of Molecular Neuroscience, v. 19, n. 1-2, p. 233-238, 2002.

SILVA, R .J. S. et al. Prevalência e fatores associados à percepção negativa da saúde em pessoas idosas no Brasil. Revista Brasileira de Epidemiologia, São Paulo, v. 15, n. 1, p. 49-62, 2012.

WORLD HEALTH ORGANIZATION. Report on the Global Tobacco Epidemic 2008: the MPOWER package. Geneva: World Health Organization, 2008. 